\title{
"Reversible splenial lesion syndrome“ (RESLES) nach einer mild verlaufenden SARS-CoV-2-Infektion
}

\section{Fallreport}

Ein 30-jähriger gebürtiger Kenianer, der als Pflegekraft in einem Altenheim arbeitet, wurde wegen einer akut aufgetretenen Vigilanzminderung, Verwirrtheit und psychomotorischer Unruhe, die ca. $1 \mathrm{~h}$ anhielten, stationär aufgenommen. Anamnestisch war 4 Tage zuvor die häusliche Quarantäne nach einer blande verlaufenden SARS-CoV-2-Infektion, die mit Fieber und Erbrechen einhergegangen war, beendet worden. Nach fremdanamnestischen Angaben der Lebensgefährtin ergab sich kein Anhalt für ein vorausgegangenes Schädel-Hirn-Trauma.

Klinisch-neurologisch zeigte sich ein verlangsamter Patient mit einer inadäquaten Reaktion auf Ansprache und psychomotorischer Unruhe. Im weiteren Verlauf klarte der Patient auf und zeigte kein weiteres fokal-neurologisches Defizit. Für einen Zeitraum von $1 \mathrm{~h}$ bestand eine retrograde Amnesie. Als begünstigender Faktor für einen möglichen epileptischen Anfall gab er einen seit dem Wochenende bestehenden Schlafmangel mit ca. 3-4h Schlaf pro Nacht an. Ein aktueller Alkohol- sowie Drogenkonsum wurden toxikologisch ausgeschlossen, jedoch war aus der Anamnese eine durch Alkoholabusus ausgelöste akute Pankreatitis 09/2020 bekannt.

Bei der Bestimmung der Serumroutinelaborparameter waren die Glutamat-Pyruvat-Transaminase mit $72 \mathrm{U} / \mathrm{l}$, die alkalische Phosphatase mit $169 \mathrm{U} / 1$ und die Lipase mit $808 \mathrm{U} / \mathrm{l}$ erhöht. Der CDT-Wert („,carbohydrate-deficient transferrin“) war mit 1,5\% (Normwert $<2,6 \%$ ) normal. Bei unauffälliger Schädelcomputertomographie (CT) zeigte das Schädel-MRT eine rundliche, in der FLAIR- und DWI-Wichtung hyperintense Läsion im hinteren Balkenanteil (Splenium), bildmorphologisch vereinbar mit einem RESLES („reversible splenial lesion syndrome").

Die Liquoruntersuchung zeigte Normalwerte für Zellzahl, Liquoreiweiß und -glukose. Es zeigte sich darüber hinaus eine isolierte, intrathekale IgG-Synthese. Das Liquorlaktat war mit $3,3 \mathrm{mmol} / \mathrm{l}$ (Normwert $<2,4 \mathrm{mmol} / \mathrm{l}$ ) erhöht. Die ergänzende Bestimmung von Thiaminpyrophosphat war mit $48 \mu \mathrm{g} / \mathrm{l}$ normal (Normwert: $40-100 \mu \mathrm{g} / \mathrm{l})$. Die Vitamin-B $\mathrm{B}_{12^{-}}(406 \mathrm{pg} / \mathrm{ml})$ und

\section{Redaktion}

A. Münchau, Lübeck

W. Pfeilschifter, Lüneburg

S. Schmidt, Bonn

DGNeurologie 2021 - 4 (6): 493-496

https://doi.org/10.1007/s42451-021-00378-8

Angenommen: 26. August 2021

Online publiziert: 24. September 2021

(c) Springer Medizin Verlag GmbH, ein Teil von Springer Nature 2021
Folsäurespiegel $(14 \mathrm{ng} / \mathrm{ml})$ waren ebenfalls normwertig. Die gesamte Serumdiagnostik und Liquorserologie inklusive Erregerdiagnostik (Lues, HIV, Hepatitis E) sowie ein Drogenscreening waren unauffällig. Elektroenzephalographisch waren keine epilepsietypischen Potenziale nachweisbar. Drei Tage später konnte der Patient nach dem erstmaligen komplex-partiellen Anfall beschwerdefrei nach Hause entlassen werden. Aufgrund der fremdanamnestischen Schilderung der Anfallssemiologie, fehlenden Hinweisen für ein SHT oder eine Intoxikation können wir das Geschehen nur als komplex-partiellen Anfall deuten. Gegen eine Enzephalopathie spricht die Kürze der Symptomatik. Die Veränderungen im Splenium waren bei einer MRTKontrolle 3 Wochen später komplett rückläufig (Abb. 1).

\section{Diskussion}

Das reversible „splenial lesion syndrome“ (RESLES) ist eine klinisch-radiologische Entität mit in der MRT reversibler, rundlicher oder diffusionsgestörter Läsion im Splenium im dorsalen Anteil des Corpus callosum (SCC) mit variablen klinischen Manifestationen [1]. Patienten mit einer reversiblen Läsion im Splenium des Corpus callosum (SCC) wurden erstmals im Jahr 1999 von Kim et al. beschrieben [2]. In der Literatur werden mindestens 3 verschiedene Begriffe für diese Entität verwendet: "mild encephalopathy with reversible splenial lesion“ (MERS, [3]), „reversible splenial lesion“ (RESLES) und „cytotoxic lesion of the corpus callosum" (CLoCC; [4-7]). Das Akronym MERS findet überwiegend bei pädiatrischen Fällen nach Virusinfekten Anwendung und unterscheidet einen Typ 1 mit isolierter Balkenläsion von einem Typ 2 mit zusätzlichen Marklagerveränderungen [8].

Im Jahr 2011 schlugen Garcia-Monco et al. anhand einer Analyse eigener und bereits publizierter Fälle das Akronym RESLES („reversible splenial lesion“) vor [9]. Dabei hoben sie die sehr gute Prognose der Betroffenen und die vielfältigen Ursachen hervor. In $60 \%$ der Fälle ist das RESLES infektassoziiert (insbesondere Influenza-A- und -B-Virus, aber auch Rotavirus, Herpesvirus-6, Epstein-Barr-Virus, Adenovirus, Mumps, CMV, Hantavirus, Salmonella enteritidis, Legionella pneumophila, Dengue-Virus, Escherichia coli, Streptococcus pneumoniae, Mycoplasma pneumoniae, Neisseria meningitidis, [9-11]). In etwa $12 \%$ wurde ein Zusammenhang mit einer Epilepsie, insbesondere nach dem abrupten Absetzen von Antikonvulsiva (Levetiracetam, Carbamazepin, Valproat) im Rahmen der prächirurgischen Diagnostik beschrieben. RESLES trat auch bei Patienten auf, bei denen Antikonvulsiva gegen neuropathische Schmerzen eingesetzt und dann abrupt pausiert worden waren. Weder die Häufigkeit der Anfälle noch ihre Art (partiell 

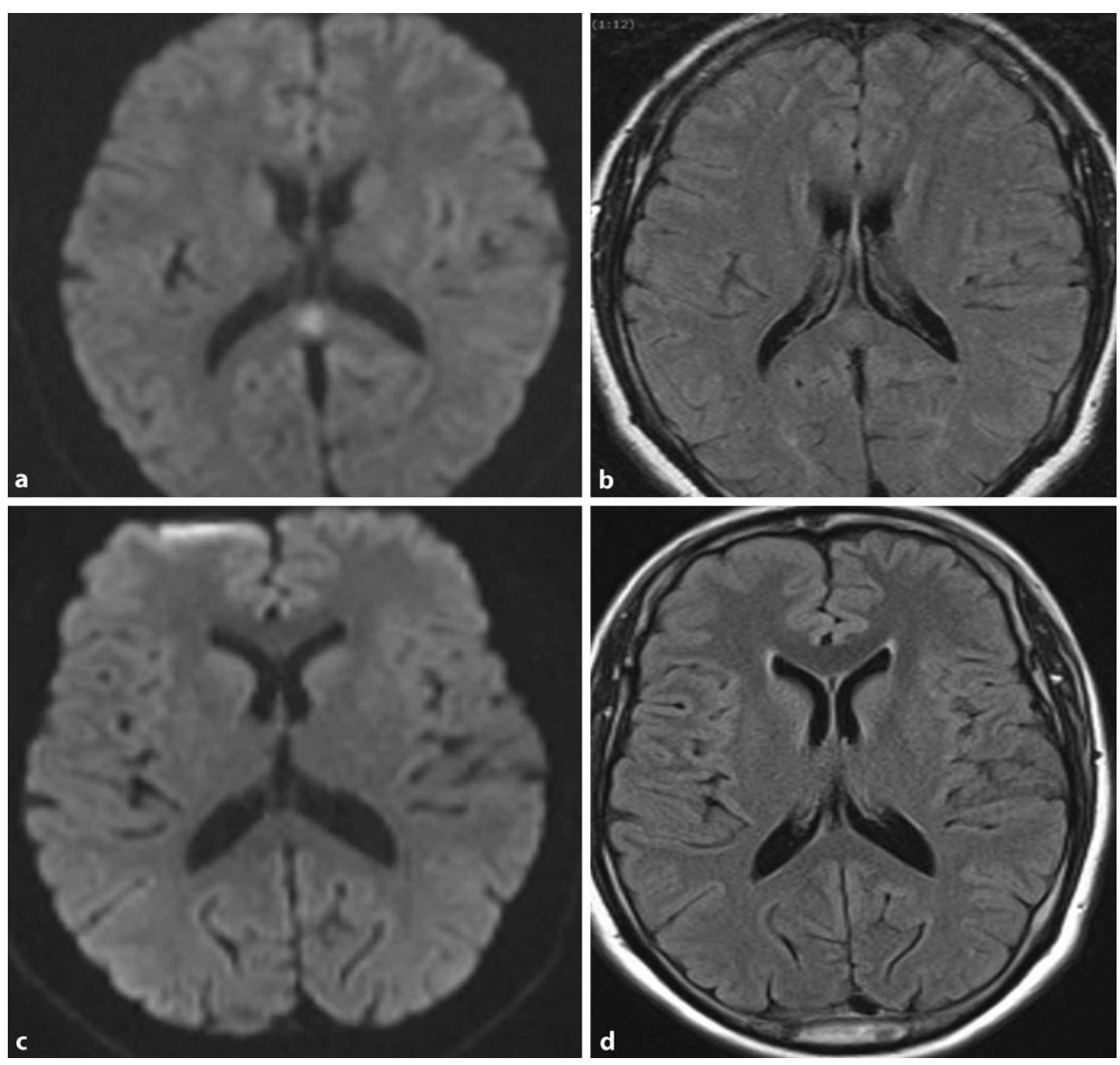

Abb. 1 MRT, DWI/FLAIR-Wichtung bei Aufnahme $(\mathbf{a}, \mathbf{b})$ und 3 Wochen später $(\mathbf{c}, \mathbf{d})$ oder generalisiert) scheinen in dieser Hinsicht relevant zu sein. In der Mehrzahl der Fälle dieser Subgruppe ist offenbar der Antikonvulsivaentzug relevanter [9]. Weiterhin finden sich in der Literatur noch weitere Ursachen wie die Einnahme von Carboplatin, Citalopram, Metronidazol, Cisplatin oder Olanzapin, Kawasaki-Syndrom, Anorexia nervosa, Höhenhirnödem (HACE), Malnutrition, ein systemischer Lupus erythematodes

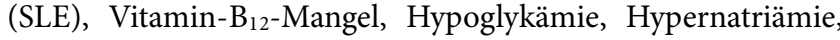
Intoxikationen, Nierenversagen, bei denen der kausale Zusammenhang offen ist [9-14]. Fast die Hälfte der RESLES Patienten bietet klinisch Zeichen einer Enzephalopathie mit normalen Liquorparametern und ohne Anzeichen einer ZNS-Infektion [9]. Bei Kindern war dies häufiger mit Bewusstseinsstörungen und Verhaltensauffälligkeiten assoziiert, sodass häufig die Bezeichnung „mild encephalopathy with reversible splenial lesion“ (MERS) verwendet wird [8]. Die Mechanismen, die RESLES zugrunde liegen, sind nicht vollständig geklärt. Signaländerungen in der DWI und ADC weisen darauf hin, dass ein passageres zytotoxisches Ödem das Korrelat der Läsionen im Splenium ist [15]. Die pathophysiologische Hypothese besagt, dass ein entzündlicher Prozess, an dem Zytokine wie IL-6 beteiligt sind, die Akkumulation von Glutamat im extrazellulären Raum auslöst, was zu einem zytotoxischen Ödem, insbesondere von Astrozyten, führt. Die selektive Anfälligkeit des Corpus callosum könnte durch seine hohe Dichte an Zytokin- und Glutamatrezeptoren erklärt werden $[4,16]$.
Seit dem ersten Auftreten von SARS-CoV-2 in der Region Hubei in China und der sich daraus entwickelten Pandemie wurde eine Reihe neurologischer Komplikationen einer SARSCoV-2-Infektion beschrieben [17, 18]. In der Literatur wurde ein Zusammenhang zwischen neurologischen Symptomen und der Schwere der respiratorischen Insuffizienz beschrieben, sodass bei Beatmungspflicht neurologische Komplikationen häufiger als bei leichter Lungenaffektion sind (45\% vs. $30 \%$; [17]). Durchschnittlich 36\% der Patienten mit COVID19 entwickeln neurologische Symptome, wie z. B. Kopfschmerzen, Bewusstseinstrübung und Parästhesien. Die SARS-CoV-2Infektion kann mit neurologischen Symptomen und Zeichen beginnen [19]. Es werden verschiedene Mechanismen diskutiert, die zu neuronalen Schäden führen können einschließlich hypoxischer Schädigungen, Zytokinsturm mit der daraus resultierenden Beeinträchtigung der Blut-Hirn-Schranke, Interaktionen mit ACE2 und akuten ischämischen Ereignissen bei Hyperkoagulabilität [20]. Ein Zusammenhang mit Spleniumveränderungen wurde in Einzelfällen sowohl bei Kindern [21] als auch bei Erwachsenen [4, 6, 7, 22-25] beschrieben. Dabei traten diese fast alle bei Patienten mit schwerer respiratorischer und neurologischer Symptomatik auf.

Der von uns vorgestellte Patient hatte bis zum Ende der Quarantäne seiner mittels PCR nachgewiesenen SARS-CoV2-Infektion weder respiratorische noch neurologische Symptome. Die typische reversible Spleniumläsion war 4 Tage nach 
Tab. 1 Zusammenstellung aller bisher veröffentlichten Kasuistiken zu Patienten, die COVID-19 assoziiert eine transiente Signalanhebung im Splenium entwickelt haben und durch neurologische Symptome auffällig wurden. Alle veröffentlichen Fälle einschließlich des in dieser Kasuistik (Hermann et al.) beschriebenen Falls waren Männer. Neben Kopfschmerzen traten häufig qualitative und/oder quantitative Bewußtseinsstörungen auf. Während sich die Mehrzahl der Betroffenen innerhalb von wenigen Tagen erholte, verstarben 2 Patienten an der COVID-19Infektion

\begin{tabular}{|c|c|c|c|c|c|}
\hline Autoren & $\begin{array}{l}\text { Alter/ } \\
\text { Geschlecht }\end{array}$ & Klinischer Befund & $\begin{array}{l}\text { SARS-CoV- } \\
\text { 2-Nachweis }\end{array}$ & $\begin{array}{l}\text { MRT: isolierte, transien- } \\
\text { te, Signalanhebung im } \\
\text { Splenium in FLAIR und } \\
\text { DWI }\end{array}$ & Verlauf \\
\hline $\begin{array}{l}\text { Agarwal } \\
\text { et al. [26] }\end{array}$ & $73 / \mathrm{M}$ & $\begin{array}{l}\text { Bewusstseinsminderung, respiratori- } \\
\text { sche Insuffizienz, Fieber }\end{array}$ & PCR & + & $\begin{array}{l}\text { Verbessert und nach } 4 \text { Wochen von } \\
\text { Intensivstation verlegt, keine Angaben } \\
\text { über weiteren Verlauf }\end{array}$ \\
\hline $\begin{array}{l}\text { Chauffier } \\
\text { et al. [27] }\end{array}$ & $47 / M$ & $\begin{array}{l}\text { Mehrtägiger Verwirrtheitszustand, } \\
\text { Kopfschmerzen }\end{array}$ & PCR & + & $\begin{array}{l}\text { Sauerstoffgabe, Entlassung nach } 7 \text { Ta- } \\
\text { gen }\end{array}$ \\
\hline $\begin{array}{l}\text { Elkhaled } \\
\text { et al. [6] }\end{array}$ & $23 / \mathrm{M}$ & $\begin{array}{l}\text { Akustische Halluzinationen, Unruhe, } \\
\text { Selbstmordgedanken, Fieber }\end{array}$ & PCR & + & $\begin{array}{l}\text { Intubation, ARDS, Nierenversagen, } \\
\text { Hepatitis, DIC, retroperitoneales Häma- } \\
\text { tom, ICB, an Tag 15. verstorben }\end{array}$ \\
\hline $\begin{array}{l}\text { De Oliveira } \\
\text { et al. [23] }\end{array}$ & $40 / \mathrm{M}$ & $\begin{array}{l}\text { Parästhesien der Extremitäten, Kopf- } \\
\text { schmerzen, Visusminderung links }\end{array}$ & PCR & + & Besserung innerhalb von 7 Tagen \\
\hline $\begin{array}{l}\text { Forestier } \\
\text { et al. [7] }\end{array}$ & $55 / \mathrm{M}$ & $\begin{array}{l}\text { Kopfschmerzen, Schwindel, Bewusst- } \\
\text { seinsstörung, hohes Fieber }\end{array}$ & PCR & + & $\begin{array}{l}\text { Verbessert und nach } 17 \text { Tagen extu- } \\
\text { biert, keine Angaben über weiteren } \\
\text { Verlauf }\end{array}$ \\
\hline $\begin{array}{l}\text { Hayashi } \\
\text { et al. [28] }\end{array}$ & $75 / \mathrm{M}$ & $\begin{array}{l}\text { Wahrnehmungsstörung, Tremor, } \\
\text { Ataxie, Urininkontinenz }\end{array}$ & PCR & + & $\begin{array}{l}\text { Neurologische Symptome bildeten } \\
\text { sich nach } 3 \text { Tagen zurück. Nach } 12 \text { Ta- } \\
\text { gen bei respiratorischer Insuffizienz } \\
\text { verstorben }\end{array}$ \\
\hline $\begin{array}{l}\text { Hermann } \\
\text { et al. }\end{array}$ & $30 / M$ & $\begin{array}{l}4 \text { Tage nach Ende der Quarantäne } \\
\text { nach blander SARS-CoV-2-Infektion: } \\
\text { komplex-partieller Anfall, agitiert, } \\
\text { Amnesie }\end{array}$ & PCR & + & $\begin{array}{l}\text { Neurologischer Befund normalisierte } \\
\text { sich innerhalb von } 24 \mathrm{~h}\end{array}$ \\
\hline $\begin{array}{l}\text { Kakadia } \\
\text { et al. [22] }\end{array}$ & $69 / M$ & $\begin{array}{l}\text { Desorientiert, gestörte Aufmerksam- } \\
\text { keit, Bradyphrenie, Fieber }\end{array}$ & $\begin{array}{l}\text { Antikörper- } \\
\text { nachweis }\end{array}$ & + & $\begin{array}{l}\text { Vollständige Rückbildung der neurolo- } \\
\text { gischen Symptome }\end{array}$ \\
\hline $\begin{array}{l}\text { Moreau } \\
\text { et al. [4] }\end{array}$ & $26 / M$ & $\begin{array}{l}\text { Akuter Verwirrtheitszustand, agitiert, } \\
\text { Fieber }\end{array}$ & $\begin{array}{l}\text { Antikörper- } \\
\text { nachweis }\end{array}$ & + & $\begin{array}{l}\text { Neurologischer Befund besserte sich } \\
\text { innerhalb von } 2 \text { Tagen }\end{array}$ \\
\hline
\end{tabular}

Fett in der vorliegenden Kasuistik erstmals beschriebener Fall

Beendigung einer häuslichen Quarantäne im klinischen $\mathrm{Zu}$ sammenhang mit einem erstmaligen komplex-partiellen Anfall aufgetreten. Zu diesem Zeitpunkt war der Patient fieberund symptomfrei. Gegen eine Enzephalopathie im Rahmen des RESLES spricht die kurze Dauer der psychopathologischen Auffälligkeiten in unserem Fall. Hingegen boten fast alle bislang publizierten Fälle mit Spleniumveränderungen schwere neurologische und pulmologische Befunde. Bemerkenswert ist auch die Tatsache, dass bei allen bislang publizierten Fällen im Erwachsenenalter (zwischen 26 und 75 Jahren) mit SARS-CoV-2Nachweis und reversibler Spleniumveränderung ausschließlich Männer betroffen waren (Tab. 1).

Zusammenfassend sollte auch bei blande verlaufenden SARS-CoV-2-Infektionen beim Auftreten neurologischer Symptome eine vollständige zerebrale magnetresonanztomographische Bildgebung erfolgen, um transiente morphologische Veränderungen nachzuweisen. In Analogie zur transienten globalen Amnesie (TGA) mit häufigem Nachweis einer kleinen punktförmigen transienten Diffusionsstörung im Hippocampus bleibt die Ätiologie der reversiblen Spleniumläsion noch offen. Hervorzuheben ist jedoch die überwiegend sehr gute Prognose des RESLES (Tab. 1).
Literatur

1. Li et al (2016) Reversible splenial lesion syndrome associated with lobar pneumonia. Medicine 95(39):e4798

2. Kim SS et al (1999) Focal lesion in the splenium of the corpus callosum in epileptic patients: antiepileptic drug toxicity? AJNR Am J Neuroradiol 20:125-129

3. Tada H, Takanashi J, Barkovich AJ et al (2004) Clinically mild encephalitis/ encephalopathy with a reversible splenial lesion. Neurology 63:1854-1858

4. Moreau A, Ego A, Vandergheynst Fet al (2020) Cytotoxic lesions of the corpus callosum (CLOCCS) associated with SARS-CoV-2 infection. J Neurol. https:// doi.org/ 10.1007/s00415-020-10164-3

5. Starkey J, Kobayashi N, Numaguchi Y, Moritani T (2017) Cytotoxic lesions of the corpus callosum that show restricted diffusion: mechanisms, causes, and manifestations. Radiographics 37(2):562-576. https://doi.org/10.1148/rg. 2017160085

6. Elkhaled W et al (2020) A 23-year-old man with SARS-coV-2 infection who presented with auditory hallucinations and imaging findings of Cytotoxic lesions of the corpus Callosum (CLOCC). Am J Case Rep 21:e928798

7. Forestier et al (2021) Cytotoxic lesion of the corpus callosum as presenting neuroradiological manifestation of COVID-2019 infection. J Neurol 268:1595-1597. https://doi.org/10.1007/s00415-020-10166-1

8. Vanderschueren Get al (2018) Pract Neurol 18:391-392

9. Garcia-Monco et al (2011) Reversible Splenial Lesion Syndrome (RESLES): What's in a Name? J Neuroimaging 21:e1-e14 
10. Lu et al (2020) Reversible Splenial lesion syndrome with some novel causes and clinical manifestations. Intern Med 59(20):2471-2480

11. Zhu Y, Zheng J, Zhang L, Zeng Z, Zhu M, Li X, Lou X, Wan H, Hong D (2016) Reversible splenial lesion syndrome associated with encephalitis/encephalopathy presenting with great clinical heterogeneity. BMC Neurol 16:49

12. Sathananthasarma P, Weeratunga PN, Chang T (2018) Reversible splenial lesion syndrome associated with denque fever: a case report. BMC Res Notes 11(1):412

13. Gao X et al (2020) Clinical analysis of reversible splenial lesion syndrome in Chinese adults. Medicine 99(36):e22052

14. Pei-lin Lu et al (2020) Reversible Splenial lesion syndrome with some novel causes and clinical manifestations. Intern Med 59:2471-2480

15. Xinying Zhang et al (2020) Reversible splenial lesion syndrome in children: clinical analysis and summary of a case series. J Int Med Res 48(4):1-9

16. Tetsuka S (2019) Reversible lesion in the splenium of the corpus callosum. Brain Behav 9(11):e1440. https://doi.org/10.1002/brb3.1440

17. SchenkM (2020) Neurologische Manifestationen: Wie COVID-19 die Nerven tangiert. Dtsch Arzteb/ 117(19):A-1001/B-843

18. Berlit P (2020) S1-Leitlinie: Neurologische Manifestationen bei COVID-19. DGNeurologie 3:495-519. https://doi.org/10.1007/s42451-020-00254-x

19. Rasmussen C, Niculescul, Patel S, Krishnan A (2020) Covid-19 involvement of the corpus callosum: potential effect of the Cytokine storm? Ajnr Am I Neuroradiol 41(9):1625-1628. https://doi.org/10.3174/ajnr.A6680

20. Wu Y, Xu X, Chen Z et al (2020) Nervous system involvement after infection with COVID-19 and other coronaviruses. Brain Behav Immun 87:18-22

21. Bektaş G, Akçay N, Boydağ K, Şevketoğlu E (2021) Reversible splenial lesion syndrome associated with SARS-CoV-2 infection in two children. Brain Dev 43(2):230-233

22. Kakadia Do B, Ahmed J, Siegal T et al (2020) Mild encephalopathy with reversible splenium lesion (MERS) in a patient with COVID-19. J Clin Neurosci 79:272-274

23. Oliveira D et al (2020) Transient lesion in the splenium of the corpus callosum associated with COVID-19. https://doi.org/10.1590/0004-282X20200183

24. Chougar et al (2020) Retrospective observational study of brain MRI findings in patients with acute SARS-coV-2 infection and neurologic manifestations. Radiology 297(3):E313-E323.https://doi.org/10.1148/radiol.2020202422

25. Sparr SA, Bieri PL (2020) Infarction of the splenium of the corpus callosum in the age of COVID-19: a snapshot in time. Stroke 51(9):e223-e226. https://doi.org/10.1161/ STROKEAHA. 120.030434

26. Agarwal N, Martini R, Pedrotti G, Sala SWD (2020) Unusual lesion in the splenium of the corpus callosum and coronavirus infectious disease-19. BJR Case Rep 6(3):20200068

27. Chauffier J et al (2020) First case of mild encephalopathy with reversible Splenial lesion in SARS-coV-2 infection. Med Mal Infect. https://doi.org/10.1016/j.medmal. 2020.09.018

28. Hayashi M, Sahashi Y, Baba Y et al (2020) COVID-19-associated mild encephalitis/ encephalopathy with a reversible splenial lesion. J Neurol Sci 415:116941 angegebenen ethischen Richtlinien. Für Bildmaterial oder anderweitige Angaben innerhalb des Manuskripts, über die Patienten zu identifizieren sind, liegt von ihnen und/oder ihren gesetzlichen Vertretern eine schriftliche Einwilligung vor.

Korrespondenzadresse

\section{Dr. R. Hermann}

Neurologische Klinik

Hegau-Bodensee-Klinikum Singen

Virchowstr. 10, 78224 Singen, Deutschland

Ruxandra.Hermann@glkn.de

\section{Einhaltung ethischer Richtlinien}

Interessenkonflikt. R. Hermann, Z. Acimov, G. Gavrilovs und C. Klötzsch geben an, dass kein Interessenkonflikt besteht.

Für diesen Beitrag wurden von den Autoren keine Studien an Menschen oder Tieren durchgeführt. Für die aufgeführten Studien gelten die jeweils dort 